\title{
The structural complexity of the dwarf galaxies of the Local Group
}

\author{
Alan W. McConnachie ${ }^{1}$, Nobuo Arimoto ${ }^{2}$ and Mike J. Irwin ${ }^{3}$ \\ ${ }^{1}$ Dept. of Physics and Astronomy, University of Victoria, Victoria, B.C., V8P 1A1, Canada \\ email: alan@uvic.ca \\ ${ }^{2}$ National Astronomical Observatory of Japan, 2-21-1 Osawa, Mitaka, Tokyo 181-8588, Japan \\ ${ }^{3}$ Institute of Astronomy, Madingley Road, Cambridge, CB3 0HA, U.K.
}

\begin{abstract}
We have undertaken a large, multi-colour, photometric, survey of distant $(D>$ $500 \mathrm{kpc}$ ) Local Group dwarf galaxies with the Subaru SuprimeCam wide field camera. These data reach to below the horizontal branch level of these systems, to a depth equivalent to earlier HST WFPC2 studies but over an area 100 times larger, making them ideal for studies of the global stellar content and structural characteristics of these systems. All eight of our targets show radial gradients and spatially distinct, multiple structural components. This implies that dwarf galaxies in general possess multiple stellar components, akin to larger galaxies. Dynamical models of these systems which do not account independently and consistently for each individual density component may be limited in their applicability.
\end{abstract}

We have used the Subaru SuprimeCam wide field camera to observed the distant Local Group galaxies Andromeda I, II, III, V, VI, VII, Cetus and DDO210 in the $V$ and $I$ bands. These observations follow on from our earlier, shallower, photometric study of some of these galaxies using the Isaac Newton Wide Field Camera (McConnachie et al. 2004, 2005, McConnachie \& Irwin 2006a,b).

The top left panels of Figure 1 are CMDs for And II, and show the quality and depth of the data obtained. These observations are a comparable depth to earlier HST WFPC2 imaging (Da Costa et al. 2000), but cover an area 100 times larger. Results for DDO210 can be found in McConnachie et al. 2006, for And II in McConnachie, Arimoto \& Irwin (2007a), and for the complete survey in McConnachie, Arimoto \& Irwin (2007b).

Every galaxy in our sample possesses radial gradients and multiple structural components. These manifest themselves through distinct density profiles for the different stellar populations present within each galaxy (the top right panel of Figure 1 shows the 1D profiles for And II, with the 2D profiles for each galaxy shown below this). This suggests that dwarf galaxies in general possess spatially distinct multiple stellar components. For at least And II, the two components possess distinct metallicities and may have different ages. It appears as if the well-known complexity in the star formation histories of these galaxies is reflected in their structural properties. The standard characterisation of the stellar structure of dwarf galaxies by a single component density profile must be seen in this light as an approximation only: dynamical models which do not account independently and consistently for each individual density component may be limited in their applicability since each component may possess distinct kinematics. This could result in misleading conclusions about the dynamical (dark matter) properties of these galaxies.

\section{References}

Da Costa, G. S., Armandroff, T. E., Caldwell, N., \& Seitzer, P. 2000, AJ, 119, 705

McConnachie, A. W., Arimoto, N., \& Irwin, M. 2007a, MNRAS, submitted 

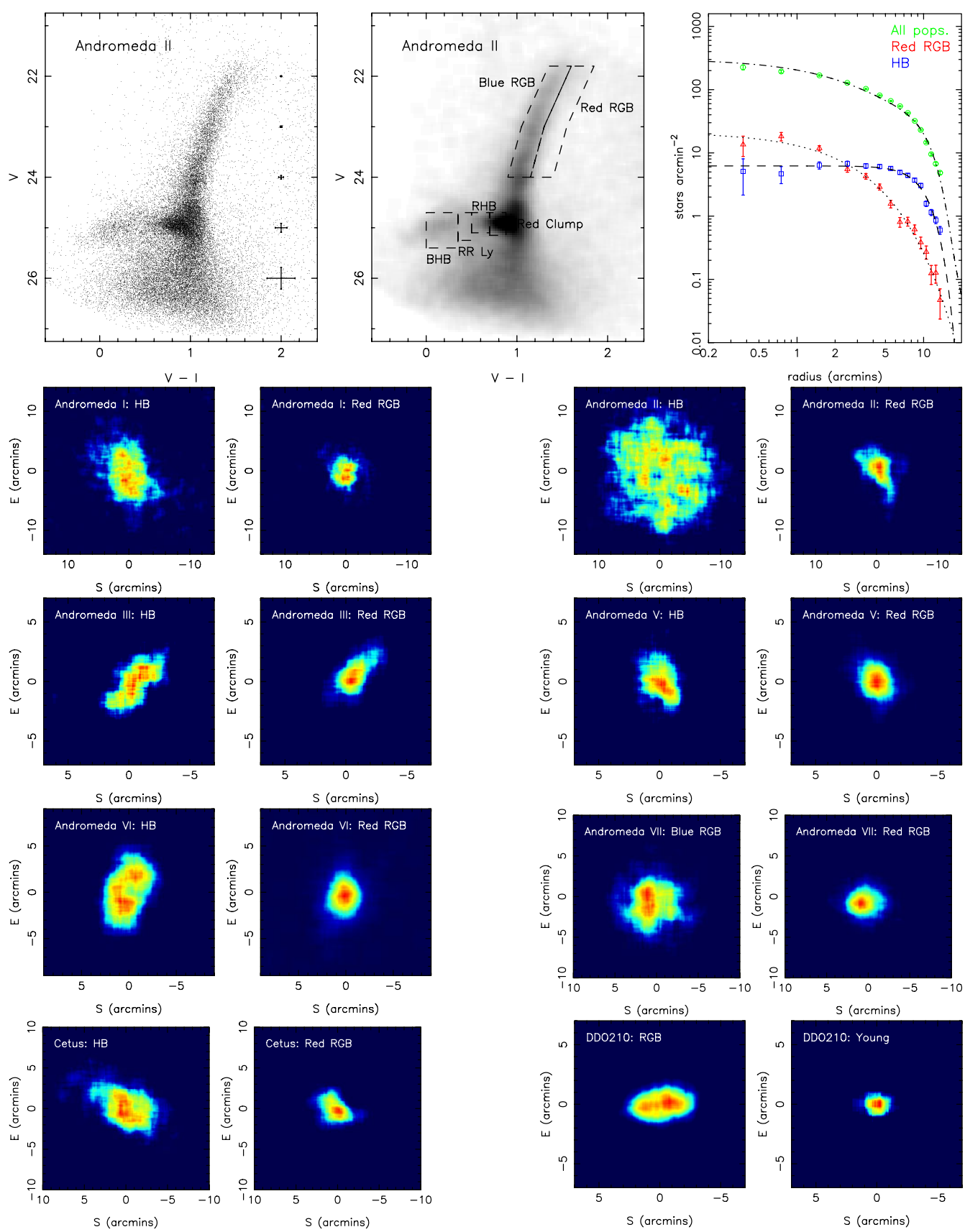

Figure 1.

McConnachie, A. W., Arimoto, N., \& Irwin, M. 2007b, ApJL, submitted McConnachie, A. W., et al., 2004, MNRAS, 350, 243

McConnachie, A. W., et al., 2005, MNRAS, 356, 979

McConnachie, A. W., Arimoto, N., Irwin, M., \& Tolstoy, E. 2006, MNRAS, 373, 715

McConnachie, A. W., \& Irwin, M. J. 2006, MNRAS, 365, 1263

McConnachie, A. W., \& Irwin, M. J. 2006, MNRAS, 365, 902 